\title{
Bi-Secting K-Means Of Document Clustering For Forensic Analysis of Computer Inspection
}

\author{
*Mr. E. YesuBabu ${ }^{1}$, Mr.J. NageswaraRao ${ }^{2}$ \\ ${ }^{I} M$. Tech in Dept. of Computer Science and Engineering, LBRCE College, Mylavaram, India. \\ ${ }^{2}$ Assistant professor in Dept. of Computer Science and Engineering, LBRCE College,Mylavaram, India.
}

\begin{abstract}
In most recent couple of decade numerous analysts investigation is anticipated to break down the criminal with that of wrongdoing. It is seen that there is a lot of acceleration in the wrongdoing rate because of the crevice between the ideal use of investigation and advances. In view of this there are numerous new accommodation for the advancement of new strategy and procedures in the field of wrongdoing examination utilizing the strategies built up on information mining, criminological, picture change over, and social mining.The vital part of computerized face off regarding is to enhance the examination of criminal exercises that include assemble, to save, investigate, advanced gadgets and give mechanical and logical statement, and to give the vital approval to experts. To consequently assemble the get archives into a rundown of important classes diverse band procedures can be utilized. Report band includes descriptor and descriptors destruction. In this paper, displays a model utilizing new mode for assessment of report bunching of criminal database by utilizing bi-secting $k$-implies grouping approach. This model exhibit the criminal information basing on the sort wrongdoing.
\end{abstract}

Fileterms: Clustering, far from being obviously true figuring, mining.

\section{Introduction}

The volume of information in the computerized world expanded from 161 hexabytes in 2006 to 988 hexabytes in 2010.this vast measure of information has an immediate effect in PC Forensics. In our specific operation area it ordinarily includes analyzing a huge number of records per PC. This movement obscure the master's capacity of examination and investigation of information. In this manner strategies for programmed information investigation. Like those broadly utilized for machine learning and information mining are of central significance. Specifically, calculations for enhancement acknowledgment from the exhortation show in content reports are propitious as it will ideally end up noticeably obvious later in the paper. The idea of package has been around for quite a while. It has a few capacity, especially with regards to data improvement and in arranging web resources. The primary reason for bunching is to find data and in the present day setting, to build up most applicable electronic assets. The examination in grouping in the long run prompted mechanized ordering , to list and in addition to recover electronic records. Bunching is a strategy in which we make group of articles that are by one means or another comparable in attributes. A definitive point of the bunching is to give a gathering of comparative records. Grouping is regularly mistaken for order, yet there is some contrast between the two. Grouping calculations are normally utilized for earlier information examination. This is totally the case in a few capacity of Computer Forensics, incorporating the one tended to in our work. From a more mechanical perspective, our datasets comprise of unlabeled articles the classes or classifications of archives that can be found are from the earlier obscure. In addition, even forward that marked datasets could be accessible from past examination. In this specific situation, the utilization of bunching calculations, which are equipped for finding inert design from content reports found in seized PCs, can value the examination performed by the master examiner.Thedigital statement is the advanced information which bolsters or negates the scene speculation [1].Documents investigation handle in PC gadget is key undertaking of the computerized easy to refute investigation prepare. Yet, this procedure of report investigation is turns out to be more intricate if the quantity of archives accessible to handle are more in number. Encourage this procedure turns out to be more unpredictable, if the span of specific store gadget increments. There are a few techniques and instruments as of now exhibited by different expert for the investigation of numerous reports. These current strategies for DFI display the numerous levels scanning approach for giving the genuine outcomes and delivering advanced confirmation that is identified with the present examination assignment. In any case, the state of such techniques is that they quit permitting the end client implies the wrongdoing agent for seeking the archives which are having a place with a particular subject in which end client consumed, or to assemble the record set in view of a given subject. In this paper, archive grouping for criminal portrayal is executed. For bunching of the info archive k-implies grouping approach is utilized. In this paper additionally utilize the information mining procedure Association Rule Mining to enhance the query output. 


\section{Related Work}

A wide collection of research has been completed in this suitable zone of the wrongdoing space. The concentration of some examiner has been put on get data from 'terms or words', declare a specific wrongdoing by utilizing name element, back of word, n-gram to enhance record grouping better and all the more enough. Concerning this, Zhiwei Li, Bin Wang, Mingjing Li, Wei-Ying Ma [12] directed an investigation in which they related back of words with name element. Their discoveries declare that the outcomes access through utilizing the name substance get to were preferable and more sufficient over those outcomes produced from information utilizing the back of word get to. Moreover, Xiang-Ying Dai, Qing-Cai Chen, XiaoLong Wang, and Jun Xu [13] enhanced Agglomerative Hierarchical Clustering by considering the consideration of the title some portion of a story. In situations where the mischance of the term was found in the title, that word was doled out as higher weight. Their discoveries demonstrated that the normal strategy was sufficient in grouping the reports of money related news. Be that as it may, the concentration of some other investigator tended to the bunching of point or occasions, though current work focus on the grouping of themes and mischance of wrongdoings. In the interim, Sheng-Tun Li, Shu-ChingKuo, Fu-Ching Tsai [14], utilized a Fuzzy Self-Organizing Map (FSOM) system to distinguish and examine the adornment of wrongdoing patterns from fleeting wrongdoing action information. Different analysts, for example, Christos Bouras and VassilisTsogkas [15], utilized grouping strategies including single, most extreme, linkage and centroid linkage progressive bunching, and also real kmeans, $\mathrm{k}$-medians and k-means++. Their discoveries declare that utilizing k-implies accomplish the best outcomes, not just at the level of residential estimation of bunching record work, additionally on genuine clients' experimentation. Moreover, when examine kmeans, single pass grouping and different methodologies of package points of news, Taeho Jo [16] uncovered that k-implies was superior to anything single pass bunching. As recommended by Zhiwei Li, Bin Wang, Mingjing Li, Wei-Ying Ma [12], evaluation of the underlying number of occasions depends, or depends on, the article counttime dissemination in their probabilistic model, where the appraisal of occasions number speaks to the underlying $(\mathrm{K})$ bunches. Notwithstanding, in this present examination, k-means and single pass bunching were connected as far as their capacity or better outcomes accomplish from breaking down the occasions of wrongdoing archives, and subsequently, assess k-implies while being utilized as a part of various subjects bigger than the underlying number of groups, and when it was utilized as a part of various topics littler than the underlying number. This was completed to differentiate its execution in the right number of introductory number of bunches, where the help of the underlying number of bunches were assembled declaration in light of this underlying number, in which it was hard to choose the underlying number of bunches and the fitting gatherings or sets of information of wrongdoing. The execution of k-implies grouping exceptionally relied on upon the underlying seed centroids. It was in this manner ordinary that this present strategy's outcome would frequently be problematic.

\section{Proposed Work}

In the proposed work a strategy for the report package for criminal distinguishing proof is accomplish. Extraction and quick data advancement or filtering.Related to information package.By utilizing NLP which a field of software engineering, manmade brainpower, and computational etymology worried with the participation amongst PCs and human (characteristic) dialects. We utilized frame NLP 1. Grammatical form Tagging - A grammatical form - especially in more present day orders, which regularly make more right refinements than the customary plan does - may likewise be known as a word class, lexical class, or lexical classification, 2. Piecing - Grouping the determine data Clustering techniques can be utilized to normally bunch the recover reports into a rundown of significant division. Record grouping includes depiction and descriptor deliberation. Descriptors are sets of words that depict the substance inside the group. Report group is by and large encouraged to be a brought together process. Case of report package is web record grouping. Figure 2 demonstrates the piece chart of the general proposed strategy. Criminal document is given as the contribution to the framework. NLP is connected on the information document to discover the things from the information files. Presently ABK implies grouping calculation is utilized to locate the comparable division from the info criminal document.ABKmeans bunching yield and NLP yield gives the yield division where a greatest thing matches.Data mining approach Association administer mining is utilized to discover the sorts of violations from the datasets, this apply on the division acquire from the grouping capacity to give the last outcome. 


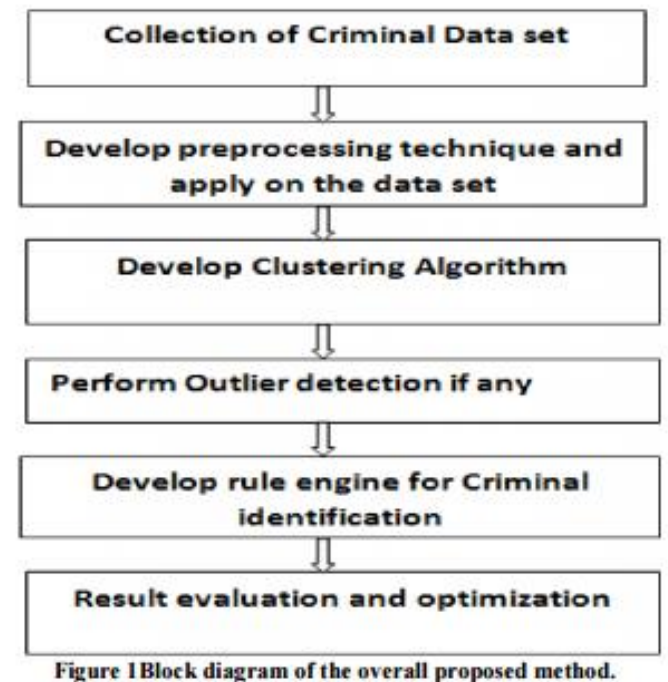

IV. Natural Language Processing

Characteristic Language (NL) preparing and comprehension of archives is an old research field with various achievements and an assortment of open issues. A portion of the examination exercises on this field are about: occasion determination (ER), sentence structure comment (GrA), data mining (IM), knowledgebase (K), naming (Lab), oddity recognition (ND), question/reply (QA), repetition diminishment (Red), semantic relatedness (SR), likeness measure (SM), rundown (Sum), literary entailment (TE), word sense disambiguation (WSD), and word sense acceptance (WSI) [20]. In the course of recent years, look into in these territories has pushed toward graphbased strategies. The decreased many-sided quality of chart strategies over vector techniques offers a more compacted and proficient idea portrayal of content. The record preparing is a most current field, which of late has being getting additional consideration because of synergistic coordination of picture and NL understanding [7]. Strategies manage archive preparing and understanding are connected with procedures, for example, division of a page(s), partition of content from pictures, picture examination and comprehension, words acknowledgment, content comprehension, relationship of content and pictures for learning revelation and portrayal, and so on.. Some of these strategies, for example, report division is exhibited, which is a "top-down" approach and delivers great outcomes under the condition that the analyzed page can be isolated into squares. Another algorithmic approach is exhibited, which is a "base up" prepare with great execution in a few classes of pages with great dispersing highlights, and "non covering" squares. Likewise, a strategy introduced that isolates pictures from content by keeping up their connections. An augmentation of the report preparing with an assortment of utilizations, for example, human PC connection (HCI), information disclosure, picture/archive databases exact recovery, and so forth is the record understanding that consolidates picture understanding-elucidation and normal dialect handling - understanding. Around 7.7\% of the reports are composed in common dialect and 5.3\% of the archives are composed in formal dialects, while rest of them are composed by utilizing different methods, them two have their benefits and faults [8]. Information is communicated in normal dialect and whatever formal procedure one may use to catch prerequisite we can't stay away from characteristic dialect, let it be utilize cases, situations and so on., as of late the work have begun to change formal to casual portrayal so the ancient rarities could be confirmed by autonomous area specialists, for example, if there should arise an occurrence of testing [9].

Clustering can be viewed as the most vital unsupervisedlearning issue happened in information digging for criminal record bunching; along these lines, all other issue of bunching is manages finding a structure in a gathering of unlabeled information A free meaning of grouping could be "the way toward arranging objects into bunches whose individuals are like each other". A group is characterized as the accumulation of items which seem to be "comparative" amongst them and are "different" to the articles having a place with different bunches. We can clarify this with an exceptionally basic case as takes after 


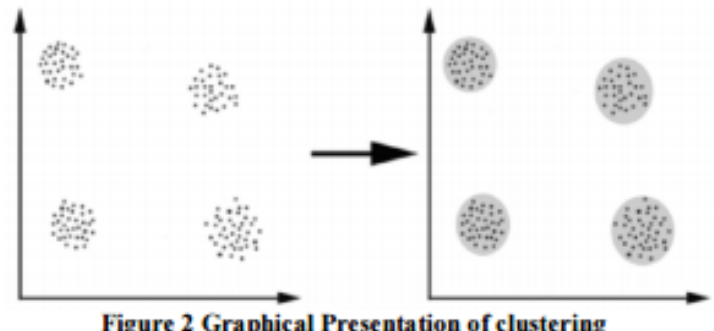

Figure 2 Graphical Presentation of clustering

In figure 1 we effortlessly recognize the 4 bunches into which the information can be partitioned; the similitude rule is remove: at least two items have a place with a similar group on the off chance that they are "close" as indicated by a geometrical separation (i.e. given separation) is known as a distancebased grouping. Reasonable grouping is another sort of bunching: at least two items have a place with a similar bunch if this one characterizes an idea regular to all articles. In another words we can state that items are gathered by their fit to graphic ideas yet noticing as per similitude measures. Thus, the objective of bunching is to decide the characteristic gathering in an arrangement of $\mathrm{n}$ marked information. In any case, how to choose what constitutes a superior bunching? It is demonstrated that there is nothing outright "best" paradigm which would be free of the last point of the grouping. Clearly, it is the client which must gives this measure, According to the necessities of the client to get the best possible outcomes [10]. There are distinctive grouping calculation proposed by various creators over the most recent couple of decade. A portion of the territories take after: 1. Versatile Bisecting KMeans Clustering Algorithm Bisecting k-Means resembles a mix of k-Means and progressive grouping. It begins with all items in a solitary group [10]. This calculation gave the consolidate approach of two algorithmKmedoidand Bisecting k-implies Algorithm The pseudo code of the calculation is shown beneath: Basic Bisecting K-implies Algorithm for discovering $\mathrm{K}$ bunches 1. Introduce: arbitrarily select $\mathrm{k}$ of the $\mathrm{n}$ information focuses as the medoids 2. Task step: Associate every information point to the nearest medoid. 3. Refresh venture: For each medoid $\mathrm{m}$ and every information point $\mathrm{o}$ related to $\mathrm{m}$ swap $\mathrm{m}$ and $\mathrm{o}$ and process the aggregate cost of the arrangement (that is, the normal uniqueness of o to every one of the information guides related toward $\mathrm{m}$ ). Select the medoid o with the most minimal cost of the design. 4. Pick a group to part 5. Discover 2 sub-bunches utilizing the essential k-Means calculation (Bisecting step) 6. Rehash step 2, the bisecting venture, for iterative circumstances and take the split that produces theclustering with the most noteworthy general comparability. 7. Rehash steps 4, 5 and 6 until the point that the coveted number of groups is come to. The basic part is which bunch to decide for part. Furthermore, there are distinctive approaches to continue. Bisecting k-implies bunching calculation can be utilized to segment the thing from the web based business webpage according to the customer interests and his past searches.A segment grouping calculation acquires a solitary parcel of the information rather than a bunching strategy, for example, the dendrogram created by a various leveled method. Segment strategies have points of interest in applications including huge informational indexes for which the development of a dendrogram is computationally restrictive. An issue going with the utilization of a segment calculation is the decision of the quantity of wanted yield groups. The parcel systems more often than not deliver bunches by streamlining a standard capacity characterized either locally (on a subset of the examples) or all around. Consolidate pursuit of the arrangement of conceivable naming for an ideal estimation of a rule is unmistakably computationally restrictive. In this manner, the calculation is normally run numerous circumstances with the best arrangement, and distinctive beginning states acquired from the greater part of the runs is utilized as the yield bunching. The most natural and every now and again utilized foundation work in segment bunching strategies is the squared blunder paradigm, which tends to function admirably with conservative and separated groups.

\section{Role Of Document Clustering In Forensic Analysis:}

PC measurable investigation includes the looking at the colossal arrangement of records. Among the greater part of that records are not important to the measurable analyst intrigue. So examining such records and reports which are out of intrigue watches out for additional tedious procedure. So to keep away from this key approach is to apply record bunching on such gigantic arrangement of documents and reports. Therefore, these record bunching gives distinctive arrangement of groups among which legal analyst break down just important archives identified with examination of revealed case. It enhances speed of the scientific investigation handle. It will likewise help for measurable inspector to break down the records and archives by just investigating illustrative of the bunches. The record bunching process includes the accompanying stages as appeared in Fig.2. 1. Gathering of Data: Collection of information includes the procedures like procuring the records and reports from the PC seized gadgets. The gathering of such records and archives includes exceptional procedures It 
incorporates diverse procedures like slithering, ordering, separating so as to gather the report. 2. Preprocessing: It comprises of steps that take as information a plain content report and yield an arrangement of tokens (which can be single terms or $\mathrm{n}$-grams) to be incorporated into the vector demonstrate. A. Tokenization: It takes message as information and yields the quantity of tokens.

\section{Conclusion}

Grouping has various applications in each field of life. We are applying this procedure whether intentionally or unconsciously in everyday life. One needs to group a great deal of thing on the premise of comparability either deliberately or unknowingly. Grouping is frequently one of the initial phases in information mining examination. The partitionedbisecting K-implies calculation likewise accomplished great outcomes when legitimately instated. Considering the methodologies for assessing the quantity of bunches, the relative legitimacy foundation known as outline has appeared to rearrangedversion.It distinguishes gatherings of related records that can be utilized as a beginning stage for investigating further connections. Also, some of our outcomes recommend that utilizing the record names alongside the report content data might be helpful for group outfit calculations. Above all, we watched that bunching calculations undoubtedly have a tendency to instigate groups shaped by either significant or unessential records, in this way adding to upgrade the master inspector's occupation. Furthermor e, our assessment of the proposed approach in five true.

\section{References}

[1] J. F. Gantz, D. Reinsel, C. Chute, W. Schlichting, J. McArthur, S. Minton, I. Xheneti, A. Toncheva, and A. Manfrediz, -The expanding digital universe: A forecast of worldwide information growth through 2010,\|l Inf. Data, vol. 1, pp. 1-21, 2007.

[2] B. S. Everitt, S. Landau, and M. Leese, Cluster Analysis. London, U.K.: Arnold, 2001.

[3] A. K. Jain and R. C. Dubes, Algorithms for Clustering Data .Englewood Cliffs, NJ: Prentice-Hall, 1988.

[4] L. Kaufman and P. Rousseeuw, Finding Groups in Gata: An Introduction to Cluster Analysis. Hoboken, NJ: Wiley -Interscience, 1990.

[5] R. Xu and D. C.Wunsch, II, Clustering. Hoboken, NJ: Wiley/IEEE Press, 2009.

[6] A. Strehl and J. Ghosh, —Cluster ensembles: A knowledge reuse framework for combining multiple partitions,॥ J. Mach. Learning Res., vol. 3, pp. 583-617, 2002.

[7] E. R. Hruschka, R. J. G. B. Campello, and L. N. de Castro, -Evolving clusters in gene-expression data,\| Inf. Sci., vol. 176, pp. 1898-1927, 2006

[8] B. K. L. Fei, J. H. P. Eloff, H. S. Venter, andM. S. Oliver, -Exploring forensic data with self-organizing maps,॥ in Proc. IFIP Int. Conf. Digital Forensics, 2005, pp. 113-123.

[9] N. L. Beebe and J. G. Clark, —Digital forensic text string searching: Improvinginformation retrieval effectiveness by thematically clustering search results, $\|$ Digital Investigation,Elsevier,vol. 4, no. 1, pp. 49-54, 2007.

[10] R. Hadjidj, M. Debbabi, H. Lounis, F. Iqbal, A. Szporer, and D. Benredjem, -Towards an integrated e-mail forensic analysis framework, „Digital Investigation, Elsevier, vol. 5, no. 3-4, pp. 124-137, 2009. self-organizing maps,\| in Proc. IFIP Int. Conf. Digital Forensics, 2005, pp. 113-123.

[11] Michael Steinbach, George Karypis, Vipin Kumar, “A Comparison of Document Clustering Techniques” at In KDD Workshop on Text Mining

[12] Zhiwei Li, Bin Wang, Mingjing Li, Wei-Ying Ma.“A Probabilistic Model for Retrospective News Event Detection”,in Proceedings of the 28th annual international ACM SIGIR conference on Research and development in information retrieval, pp. 106-113, 2005.

[13] Dai X, Chen Q, Wang X,Xu J. "Online topic detection and tracking of financial news based on hierarchical clustering," in Proceeding of International Conference on Machine Learning and Cybernetics, pp. 3341-3346, 2010.

[14] Sheng-Tun Li, Shu-ChingKuo, Fu-ChingTsai."An intelligent decision-support model using FSOM and rule extraction for crime prevention”, Expert Systems with Applications, Elsevier, Vol (37), no. 10, PP. 7108-7119, 2010.

[15] Bouras C, Tsogkas V. "Assigning Web News to Clusters," in Proceedings of Conference on Internet and Web Applications and Services, pp. 1-6, 2010.

[16] TaehoJo.“Clustering News Groups using Inverted Index based NTSO,’NDT, First International Conference on Networked Digital Technologies, PP. 1-7, 2009.

[17] Aouf M, Lyanage L, Hansen S. "Review of data mining clustering techniques to analysedata with high dimensionality as applied in gene expression data (June 2008)" in Proceeding of International Conference on Service Systems and Service Management, pp. 1-5, 2008

IOSR Journal of Computer Engineering (IOSR-JCE) is UGC approved Journal with Sl. No. 5019, Journal no. 49102.

Mr. E. YesuBabu. "Bi-Secting K-Means Of Document Clustering For Forensic Analysis of Computer Inspection." IOSR Journal of Computer Engineering (IOSR-JCE) 19.4 (2017): 78-82. 